\title{
Study of preferential solvation in binary solvent mixtures by the spectro-streak picosecond technique
}

\author{
N.Kh. Petrov ${ }^{1}$, A. Wiessner, T. Fiebig, H. Staerk * \\ Max-Planck Institut für biophysikalische Chemie, Abteilung Spektroskopie und Photochemische Kinetic, Am Fassberg, \\ D-37077 Göttingen, Germany
}

Received 21 March 1995; in final form 22 May 1995

\begin{abstract}
Using the picosecond spectro-streak method we monitored the dynamics of preferential solvation of a charge-transfer dipole in binary mixtures of solvents strongly different in dielectric constant. The observed slow $(0.25-1 \mathrm{~ns}) \mathrm{spectral}$ red-shift of the charge-transfer (exciplex) fluorescence is considered to be mainly due to the formation and the dielectric enrichment of the solvation shell around the dipolar solute molecules by diffusion of polar molecules from the bulk of the solvent mixture.
\end{abstract}

\section{Introduction}

Although preferential solvation in binary solvent mixtures has been of interest for decades [1] it is not yet understood completely. Solvatochromic shifts of fluorescence spectra associated with this physicochemical phenomenon have been extensively studied under steady-state conditions (see, for example, Ref. [2]). A previous time-resolved study of spectral shifts of exciplex fluorescence in binary solvents, components of which were greatly different in dielectric constant, was carried out with small concentrations of the polar component. Small concentrations were

\footnotetext{
${ }^{1}$ Semenov Institute of Chemical Physics, Russian Academy of Sciences, 117344 Moscow, Russian Federation.

* Corresponding author.
}

necessary because of a low time resolution (on the nanosecond scale) of the apparatus [3]. The recent development of picosecond time- and wavelength-resolved spectroscopy allows the study of preferential solvation in binary mixtures under more realistic conditions. An instrument developed in this laboratory based on a single-shot spectro-streak apparatus $[4,5]$ is especially convenient for this purpose.

One of the physico-chemical aspects of preferential solvation in binary mixtures is the dielectric enrichment of the solvent shell around the excited solute species by molecules of the polar component. This process is generally considered to be determined by a solute-solvent dielectric interaction alone [2].

The purpose of this Letter is to provide new experimental data on the problem of preferential solvation in binary mixtures obtained on a picosec- 
ond time scale and to discuss them in the framework of a simple diffusion model.

\section{Experimental}

To monitor preferential solvation effects a molecule is required as a fluorescence probe which, upon excitation, rapidly reaches an excited chargetransfer (CT) state with a large dipole moment, sufficient CT-fluorescence quantum-yield and a long CT lifetime. We used the compound $3^{\prime}-\mathrm{N}, \mathrm{N}$-dimethylamino-3H-4,5-benzonaphthantracene (Fig. 1). This constitutes pyrene rigidly coupled to N,N-dimethylaniline (DMA). The spectroscopic and dynamic properties of this compound (Py(S)DMA) in different neat solvents have been extensively investigated [5-7]. The long fluorescence lifetime (25-29 ns) of the CT-state $\left(\mathrm{Py}^{-}(\mathrm{S}) \mathrm{DMA}^{+}\right)^{*}$ and the large CT dipole moment, estimated to be about 20-25 D, make this compound a suitable candidate as a probe for dielectric enrichment studies, in that the time available to reach a steady-state dielectric enrichment around the CT dipole in binary solvents is not much limited by the lifetime of the excited CT-state. The steady-state fluorescence spectra of Py(S)DMA $\left(3 \times 10^{-5} \mathrm{M}\right)$ were measured with a Perkin-Elmer LS-50 spectrofluorimeter. To observe time-resolved fluorescence spectra of the CT species we used the spectro-streak apparatus described in detail previously $[4,8]$. The spectro-streak photometer measures fluorescence intensity, time, and wavelength, $I(t, \lambda)$, with a single picosecond excitation pulse simultaneously. It consists of a picosecond Nd:YAG or Nd:glass laser (pulse width $25-30$ ps or 7 ps, respectively) and a single-shot streak camera (Hamamatsu C1370-01) equipped with a grating objective [4]. From the set of time-evolving spectral series obtained with the spectro-streak method we extracted the Stokes shift correlation function $C(t)$.

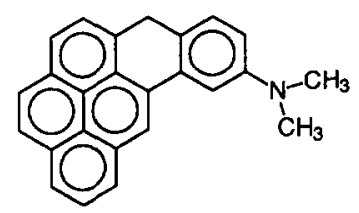

Fig. 1. Scheme of the probe molecule Py(S)DMA.
We adapted the method of analysis applied by Maroncelli and Fleming [9]. There, the time evolution of the band is determined by fitting the time-resolved spectra to a common 'log-normal' lineshape function [10]. The position of the maximum $\tilde{\nu}_{\mathrm{p}}(t)$ as a function of time is finally fitted to a sum of exponentials according to

$$
\begin{aligned}
& C(t)=\tilde{\nu}_{\mathrm{p}}(t)-\tilde{\nu}_{\mathrm{p}}(\infty)=\sum_{i=1}^{n} A_{i} \exp -\left(t / \tau_{i}\right) \\
& \text { with } \sum_{i=1}^{n} A_{i} \leqslant \tilde{\nu}_{\mathrm{p}}(0)-\tilde{\nu}_{\mathrm{p}}(\infty) .
\end{aligned}
$$

The solvents used in the present investigation, toluene, chlorobenzene, tetrahydrofurane (THF), dimethylsulfoxide (DMSO), dimethylformamide (DMF) and acetonitrile (ACN), were of spectroscopic grade (Uvasol, Merck) and used as received. Samples were saturated with nitrogen before measurements, which were carried out at room temperaturc. The diclectric constants of the mixtures were measured with the capacitor method. The density plot $\rho(x)$ as a function of the mole fraction $x$ of the polar compound of the mixtures for $x=0$ to $x=1$ followed a straight line.

\section{Results and discussion}

On increasing the mole fraction, e.g. $0.05-0.26$ mol\% of the polar component of the solvent mixtures studied, we observed red-shifts of the fluorescence-probe charge-transfer spectra: first a rapid red-shift (on a picosecond time scale, and not resolved in the spectro-streak evaluated in Fig. 2) followed by a relatively slow shift further to longer wavelengths. The characteristic time of the slow shifts is $\approx 0.5 \mathrm{~ns}$ and $\Delta \tilde{\nu} \geqslant 2000 \mathrm{~cm}^{-1}$ for mixtures of $x=0.05$ to 0.13 ; this corresponds approximately to concentrations in the order of $1 \mathrm{~mol} / 1$ of the polar component. In this range of the polar fraction the slow process is observed for all mixtures studied (i.e. toluene /DMSO, toluene/DMF, and toluene / ACN) although in the case of the toluene/DMSO mixture it seems most pronounced. Generally, the more polar the solution, the greater the shift. Typical time-resolved spectra are shown in Fig. 2. For neat solvents, 

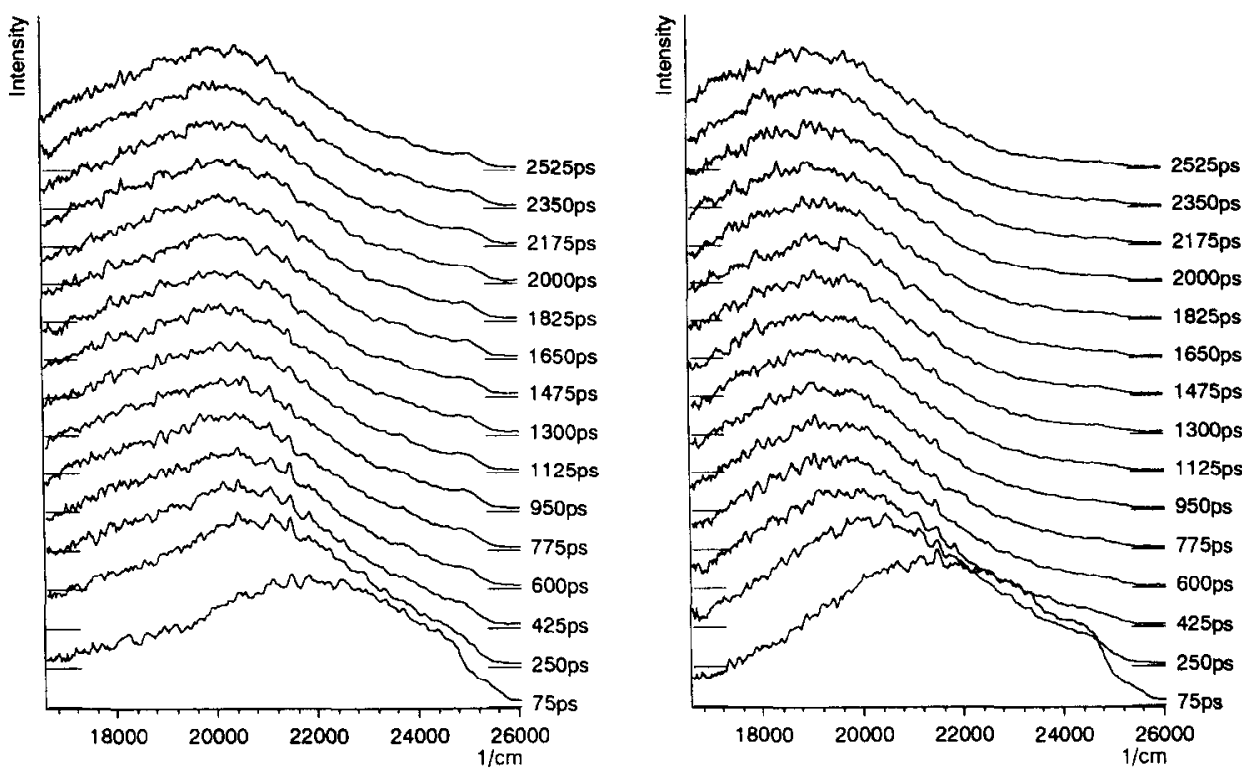

Fig. 2. Time-resolved excited state spectra of Py(S)DMA in solvent mixtures. Left: in toluene/DMF(9 mol\%), right: in toluene/DMSO(9 mol\%).

such as THF or chlorobenzene, the slow feature is absent (see Fig. 3). It is worth noting that the dielectric constant of chlorobenzene $(\epsilon=5.6)$ is approximately equal to that of the binary mixtures in which the slow spectral component exists (e.g.

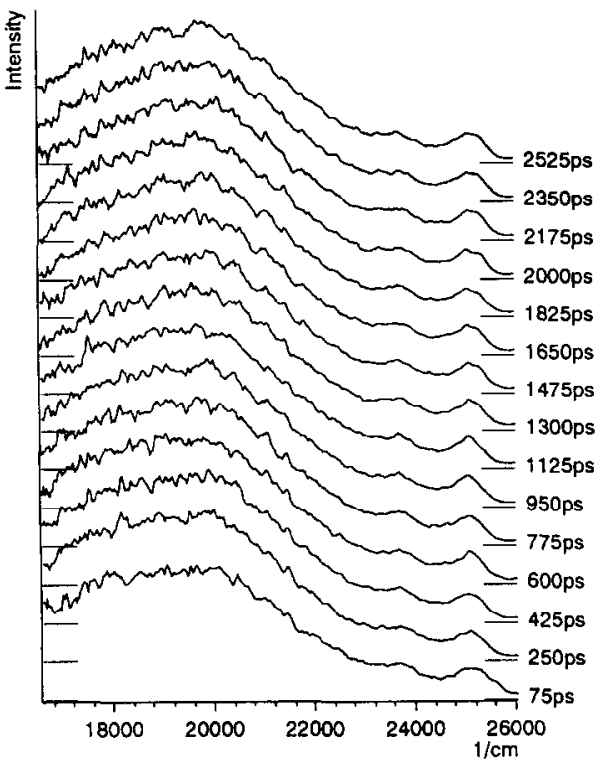

toluene/DMSO $(x=0.09))$. The steady-state CTfluorescence spectra of $\left(\mathrm{Py}^{-}(\mathrm{S}) \mathrm{DMA}^{+}\right)^{*}$ nearly coincide with the time-resolved spectra averaged over a time interval between 4 and 6 ns (see, for example, Fig. 4).

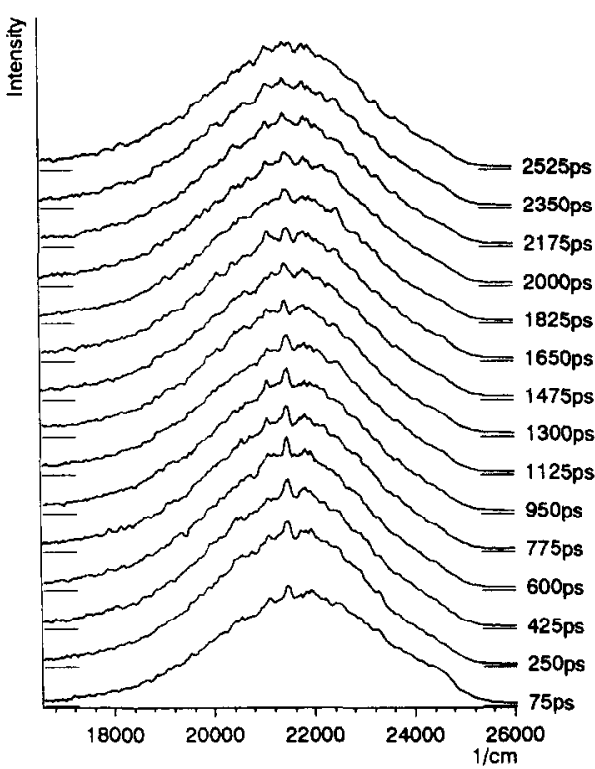

Fig. 3. Time-resolved excited state spectra of Py(S)DMA in neat solvents. Left: in THF, right: in chlorobenzene. 
The observed dynamic Stokes shifts in the binary solvents are explained as follows. After excitation of the pyrenyl moiety of the supermolecule, Py * (S)DMA, to the first excited singlet state, photoinduced intramolecular electron transfer takes place which brings about a significant increase in the dipole moment. The initial red-shift of the CT-fluorescence observed on a fast picosecond or femtosecond time scalc is mainly duc to the reorientation of the (few) polar solvent molecules or clusters initially present in the immediate vicinity of the fluorescent probe molecules $\left(\mathrm{Py}^{-}(\mathrm{S}) \mathrm{DMA}^{+}\right)^{*}$. The system reaches a 'first' equilibrium of the CT state. For our present topic, however, the mechanism of producing this initial spectral shift, the orientational relaxation, is not of primary interest (e.g. the experimental solvation time of DMSO has been determined as $\tau_{\mathrm{S}} \approx 1.2 \mathrm{ps}$ [11]). The subsequent slow CT-band shift towards the final equilibrium, on a time scale of a few hundred picoseconds to nanoseconds, is attributed to the dynamics of the dielectric enrichment process. The time for the 'local phase separation', i.e. the time for the further growth of the solvation shell, depends on the concentration of polar component molecules and is on a nanosecond time scale (as can be estimated applying simple diffusion calculations), in agreement with the experimental results.

The effective average dielectric constant $\epsilon_{\text {eff }}$ of the enriched and relaxed shells is thus different from the bulk dielectric constant $\epsilon \equiv \epsilon_{\text {mix }}$ of the mixture as measured with the capacitor method. The usual plot of $\tilde{\nu}_{\max }$ (the maximum of the CT-fluorescence band) against the Onsager polarity function [12] $f_{0}(\epsilon)=(\epsilon-1) /(2 \epsilon+1)$ or the Debye polarity function $f_{\mathrm{D}}(\epsilon)=(\epsilon-1) /(\epsilon+2)$ is therefore rather pointless in our case, whereas $\epsilon_{\text {eff }}$ is not directly measurable. A plot of $\Delta \tilde{\nu}_{\max }$ (relaxed) against the mole fraction $(x)$ of the polar component shows the anticipated saturation effect. Table 1 provides the measured data, while Fig. 5 shows the plot of $\tilde{\nu}_{\max }$ versus $x$ (DMSO) for $\left(\mathrm{Py}^{-}(\mathrm{S}) \mathrm{DMA}^{+}\right)^{*}$ in a toluene/DMSO mixture as obtained from photostationary CT-fluorescence measurements. Table 1 also contains the measured values for $\epsilon(x)$ and $n(x)$ as well as the calculated solvent parameters $\Delta f_{\text {mix }}=f_{0}$ $-\frac{1}{2} f^{\prime}$, with $f^{\prime}=\left(n^{2}-1\right) /\left(2 n^{2}+1\right)$ for the mixtures in the range $x=0.04-0.27$ DMSO.

Beyond the first solvation shell, which is assumed to be formed by random-walk diffusion, a characteristic radius $r_{0}$ for field-induced diffusion in the inhomogeneous dipole field may be defined. It can be obtained approximately by setting the dipoledipole interaction energy equal to the energy of thermal motion: $\mu_{\mathrm{M}} \mu_{\mathrm{S}} / 4 \pi \epsilon_{0} \epsilon r^{3}=k_{\mathrm{B}} T$. For $\left(\mathrm{Py}^{-}(\mathrm{S}) \mathrm{DMA}^{+}\right)^{*}$ with dipole moment $\mu_{\mathrm{M}} \approx 20 \mathrm{D}$ [5] and $\mu_{\mathrm{S}}(\mathrm{DMSO}) \approx 4 \mathrm{D}$ we obtain $r_{0} \approx 7.3 \AA$ at

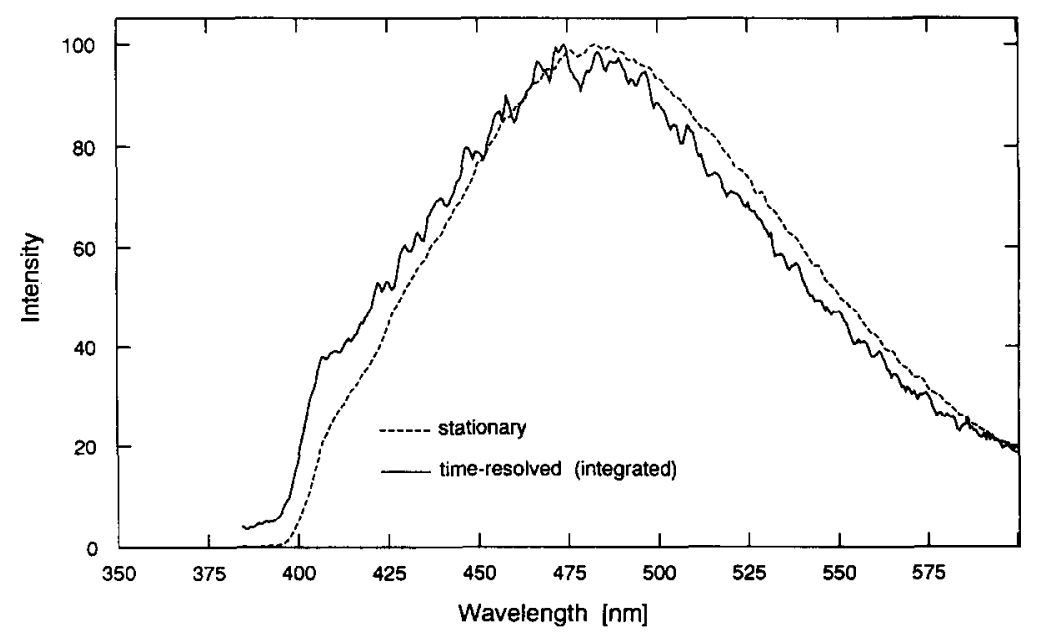

Fig. 4. Steady-state fluorescence spectra (full line) of ( $\left.\mathrm{Py}^{-}(\mathrm{S}) \mathrm{DMA}^{+}\right)^{*}$ and averaged over a time range of $6 \mathrm{~ns}$ (broken line). Solvent mixture: toluene and 4 mol\% DMSO. 
Table 1

Experimental solvent mixture parameters and fluorescence maxima

\begin{tabular}{lllll}
\hline$x$ (DMSO) & $\epsilon$ & $n$ & $f_{0}-\frac{1}{2} f^{\prime}$ & $\bar{\nu}_{\max }\left(\mathrm{cm}^{-1}\right)$ \\
\hline 0.042 & 3.26 & 1.4949 & 0.1876 & 20320 \\
0.050 & 3.43 & 1.4938 & 0.1964 & 19985 \\
0.062 & 3.69 & 1.4938 & 0.3083 & 19620 \\
0.069 & 3.84 & 1.4938 & 0.2143 & 19395 \\
0.086 & 4.23 & 1.4937 & 0.2287 & 19060 \\
0.099 & 4.54 & 1.4936 & 0.2385 & 18785 \\
0.111 & 4.85 & 1.4935 & 0.2471 & 18670 \\
0.135 & 5.48 & 1.4934 & 0.2618 & 18400 \\
0.160 & 6.15 & 1.4931 & 0.2746 & 18195 \\
0.186 & 6.92 & 1.4929 & 0.2863 & 18000 \\
0.196 & 7.24 & 1.4928 & 0.2905 & 17860 \\
0.222 & 8.06 & 1.4925 & 0.2999 & 17720 \\
0.275 & 9.94 & 1.4917 & 0.3158 & 17470 \\
\hline
\end{tabular}

room temperature for $\epsilon \approx 5$. The fact that the interaction energy is actually a function of distance and angle coordinates is ignored in this approximation.

Note that in a volume of $r_{0}^{3} \approx 3.9 \times 10^{-22} \mathrm{~cm}^{3}$ there are only $r_{0}^{3} c_{\text {DMSO }}=3.9 \times 10^{-22} \cdot 6 \times 10^{20}$ $\mathrm{cm}^{-3} \approx 0.23$ particles for a DMSO concentration (particle density $c_{\text {DMSO}}$ ) of $1 \mathrm{~mol} / \mathrm{l}=6 \times 10^{20}$ $\mathrm{cm}^{-3}$. This means that in the vicinity of a Py(S)DMA molecule only a few DMSO molecules are located on average prior to the photoinduced formation of the electronically excited dipolar exciplex (unless some kind of initial formation of complexes between Py(S)DMA in the ground state and polar DMSO molecules or DMSO clusters in the toluene solution exist). Therefore, we assume the observation of the growth and the completion of the first solvation shell

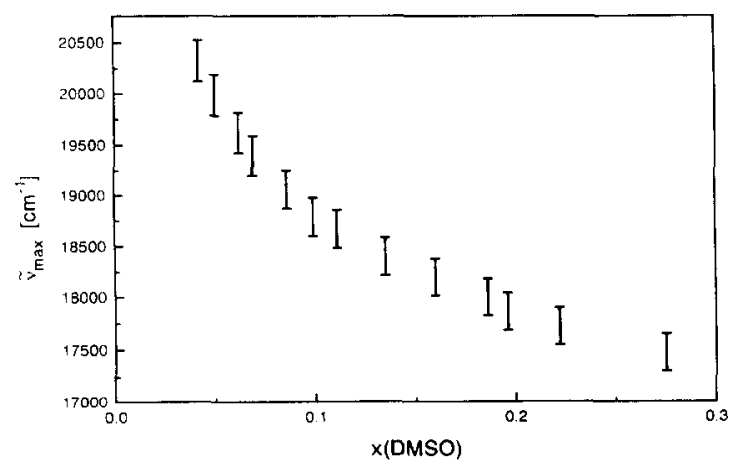

Fig. 5. Final values of the CT-fluorescence band maxima of Py(S)DMA versus mole fraction $(x)$ of DMSO in a toluene/DMSO mixture. around Py(S)DMA predominantly by free diffusion of DMSO molecules from the bulk of the solution. From our spectro-streak measurements, we obtained for the above system with mole fractions $x$ (DMSO) the following mean relaxation times $\langle\tau\rangle: 0.25 \mathrm{~ns}$ $(x=0.09), 0.45 \mathrm{~ns}(x=0.05), 0.9 \mathrm{~ns}(x=0.02)$. For more dilute solutions of a polar solvent the enrichment equilibrium time tends towards a limit which is a few nanoscconds in liquids of low viscosity.

In earlier experiments with anthracene- $\left(\mathrm{CH}_{2}\right)_{n}-$ DMA in binary mixtures of ethylacetate/acetonitrile we measured the complicated exciplex fluorescence decay functions at single wavelengths [13]. The individual data after proper weighting could be assembled - though with difficulties - to yield time-resolved spectral information, such as the dynamics of bandshifts. These earlier experiments and experiences gained in our laboratory led to the development of the spectro-streak apparatus [4] which allows the easy and correct evaluation of kinetic parameters in the presence of dynamic band shifts.

Supplementary to the above explanation based on dielectric enrichment due to free diffusion and to a lesser extent due to the influence of the inhomogeneous dipole field, we are presently exploring the question to which extent the final size of the polar solvent shell or cluster may be additionally determined by surface tension (surface between the two phase-separated components). A relation to the classical theory of nucleation [14] may be established. Nucleation in binary mixtures has been discussed in connection with radical ion pair recombination reactions in the presence of external magnetic fields [15].

Solvatochromic probes in a variety of ionic solutions have been studied in recent experimental and theoretical work [16-18]. Those authors describe the spectral changes as a function of added salt or carry out numerical simulations of solvation dynamics in electrolyte solutions by establishing the ionic atmosphere solvation function. Future theoretical treatments of solvation dynamics in binary nonionic solvents may benefit from that work.

\section{Acknowledgement}

The fluorescence probe compound was synthesized by Dr. W. Kühnle in this labonatory. The 
authors thank B. Frederichs and H. Meyer for technical assistance. NKhP thanks the Max-Planck-Society and the Russian Fund for Fundamental Researches for support.

\section{References}

[1] C. Reichardt, Solvent effects in organic chemistry (Verlag Chemie, Weinheim, 1979) ch.2.

[2] P. Suppan, J. Chem. Soc. Faraday Trans. I 83 (1987) 495.

[3] G.S. Beddard, S.E. Carlin and C. Lewis, J. Chem. Soc. raraday Trans. II, 71 (1975) 1894.

[4] A. Wiessner and H. Staerk, Rev. Sci. Instrum. 64 (1993) 3430.

[5] A. Wiessner, Dissertation, Universität Göttingen (1994).

[6] G. Hüttmann, W. Kühnle, H. Staerk and A. Wiessner, Book of Abstracts XV. Intern. Conf. on Photochem., Paris, 1991.
[7] A. Wiessner, G. Hüttmann, W. Kühnle and H. Staerk, submitted for publication.

[8] H. Staerk, A. Wiessner and W. Kühnle, J. Fluorescence 4 (1994) 87.

[9] M. Maroncelli and G.R. Fleming, J. Chem. Phys. 86 (1987) 6221.

[10] P.B. Siano and D.E. Metzler, J. Chem. Phys. 51 (1969) 1856.

[11] W. Jarzeba, G.C. Walker, A.E. Johnson and P.F. Barbara, Chem. Phys. 152 (1991) 57.

[12] L. Onsager, J. Am. Chem. Soc. 58 (1936) 1486.

[13] A. Helmbold, Diploma Thesis, Universität Göttingen (1988).

[14] J. Frenkel, Kinetic theory of liquids (Dover, New York, 1955).

[15] N.Kh. Petrov, V.N. Borisenko and M.V. Alfimov, J. Chem. Soc. Faraday Trans. 90 (1994) 109.

[16] C.F. Chapman and M. Maroncelli, J. Phys. Chem. 95 (1991) 9095.

[17] E. Bart and D. Huppert, Chem. Phys. Letters 195 (1992) 37.

[18] E. Neria and A. Nitzan, J. Chem. Phys. 100 (1994) 3855. 